\title{
Recomendaciones para el manejo de la cardiotoxicidad relacionada con el tratamiento del cáncer. Segunda parte
}

\author{
Coordinadora: Andreina Gómez ${ }^{1}$ \\ Comité de redacción: Enrique Soto ${ }^{2}$, Andreina Gómez, Rosanna Torighelli
}

\begin{abstract}
Resumen
Las nuevas terapias oncológicas han logrado aumentar la sobrevida del paciente con cáncer, observando, sin embargo, un incremento de la morbilidad y mortalidad vinculadas a sus efectos secundarios. El desarrollo de eventos cardiovasculares adversos impacta negativamente en el pronóstico durante el tratamiento del cáncer, pero también en los supervivientes al cáncer, donde las enfermedades cardiovasculares (ECV) y las segundas neoplasias son la principal causa de muerte ${ }^{(1-5)}$.

La cardiotoxicidad inducida por el tratamiento del cáncer se define como el conjunto de ECV derivadas de los tratamientos oncológicos. Su manifestación es variada e incluye el desarrollo de disfunción ventricular, insuficiencia cardíaca (IC), isquemia miocárdica, hipertensión arterial (HTA) y arritmias, entre otras. Puede ser consecuencia tanto del efecto directo del tratamiento sobre la estructura y función cardíacas, como del desarrollo acelerado de enfermedad cardiovascular ${ }^{(6-9)}$.

Con frecuencia, se utiliza el término cardiotoxicidad como sinónimo de disfunción ventricular por quimioterapia (DV-QT). Dado que la cardiotoxicidad abarca un espectro más amplio de afectación cardiovascular, creemos conveniente hablar de DV-QT para referirnos a la afectación de la función sistólica del ventrículo izquierdo.

La DV-QT y el desarrollo de IC representan una de las complicaciones más temidas por su impacto pronóstico en la esfera cardiovascular y oncológica, dado que limitan el arsenal terapéutico para el tratamiento del cáncer ${ }^{(5,10)}$.

Han sido creadas diversas sociedades de cardio-onco-hematología con el fin de generar recomendaciones de práctica clínica y formar profesionales capacitados para el manejo de las complicaciones CV del tratamiento del cáncer ${ }^{(11)}$.

La cardio-oncología es una disciplina en creciente y continuo desarrollo. Creemos que es fundamental realizar tareas de formación médica continua, así como también estimular el trabajo conjunto de diversas especialidades para brindar una mejor asistencia.

Este texto es el resultado del trabajo de un equipo multidisciplinario que incluye cardiólogos, hematólogos y oncólogos, y pretende brindar información a los integrantes del equipo de salud involucrados en la asistencia de pacientes oncológicos. Debido a la extensión del presente texto, hemos decidido fraccionar el contenido en tres partes para facilitar su difusión.
\end{abstract}

Palabras clave: QUIMIOTERAPIA

CÁNCER

CARDIOTOXICIDAD

CARDIO-ONCOLOGÍA

DISFUNCIÓN VENTRICULAR

INSUFICIENCIA CARDÍACA

DETECCIÓN PRECOZ

SEGUIMIENTO

1. Cátedra de Cardiología, Unidad de Cardio-Oncología, Hospital de Clínicas. Montevideo, Uruguay.

2. Servicio de Cardiología, CASMU-IAMPP. Montevideo, Uruguay.

3. Servicio de Cardiología, Instituto Nacional del Cáncer, ASSE. Montevideo, Uruguay.

Correspondencia: Dra. Andreina Gómez. Correo electrónico: andreinagomezeli@gmail.com

Los autores declaran no tener conflictos de intereses.

Recibido Set 24, 2021; aceptado Oct 29, 2021 


\section{Recommendations for the management of cardiotoxicity related to cancer treatment. Part two}

\section{Summary}

New oncological therapies have been successful in increasing cancer patient survival, but they have also led to an increase in morbidity and mortality linked to their side effects. During cancer treatment, the development of cardiovascular side effects has a negative impact in prognosis, but also in cancer survivors, in whom cardiovascular diseases and secondary malignancies are the main cause of death.

Cancer related cardiotoxicity is defined as the development of cardiovascular diseases related to cancer treatment. Clinical presentation is broad involving ventricular dysfunction, heart failure, myocardial ischemia, arterial hypertension and arrhythmias among others. This may result from the direct cardiovascular effect of a cancer treatment or accelerated development of cardiovascular diseases.

Frequently, in the literature cardiotoxicity and chemotherapy related ventricular dysfunction are used as synonyms. However, cardiotoxicity includes a broad spectrum of cardiovascular manifestations, thus in this text we refer to chemotherapy related ventricular dysfunction as the presence of left ventricular systolic impairment.

Chemotherapy related ventricular dysfunction and heart failure are two of the most feared complications of cancer treatment due to its impact on cardiovascular and oncological prognosis, affecting treatment options.

Numerous worldwide cardio-onco-hematology societies have emerged to generate clinical practice guidelines and improve the diagnosis and evaluation of cardiovascular cancer treatment side effects.

Cardio-Oncology is a discipline in continuous growth and development. We strongly believe that continuum medical education and a multidisciplinary approach is necessary to provide a quality health care.

This text is the result of a multidisciplinary work involving cardiologists, hematologists and oncologists. It is our goal to provide information to the health care team involved in the assistance of cancer patients. Due to its extension, it will be divided in three parts.

Key words: $\quad$ CHEMOTHERAPY

CANCER

CARDIOTOXICITY

CARDIO-ONCOLOGY

VENTRICULAR DYSFUNCTION

HEART FAILURE

EARLY DETECTION

FOLLOW-UP

\section{Recomendações para a gestão de cardiotoxicidade relacionada com o tratamento do câncer. Primeira parte}

\section{Resumo}

O desenvolvimento de novas terapias oncológicas levou a um aumento na sobrevida dos pacientes, mas ao mesmo tempo traz consigo morbidades relacionadas aos tratamentos. O desenvolvimento de efeitos cardiovasculares adversos tem um impacto negativo no prognóstico dos pacientes em tratamento, bem como nos pacientes considerados curados, nos quais doença cardiovascular e malignidades secundárias são as principais causas de morte.

Cardiotoxicidade relacionada ao câncer é definida como o desenvolvimento de doença cardiovascular secundária ao tratamento. A gama de apresentações clínicas é ampla, podendo se manifestar como disfunção ventricular, insuficiência cardíaca, isquemia miocárdica, hipertensão arterial, arritmias, entre outras. Isto pode ser resultante de desenvolvimento e progressão acelerados de doença cardiovascular ou por efeito direto das terapias.

Frequentemente é dito na literatura que cardiotoxicidade e disfunção ventricular relacionada à quimioterapia são sinônimos. Entretanto, cardiotoxicidade engloba um amplo espectro de manifestações cardiovasculares. Neste texto, portanto, nos referimos à disfunção ventricular causada por quimioterápicos exclusivamente como a presença de disfunção sistólica ventricular esquerda.

Disfunção ventricular relacionada à quimioterapia e insuficiência cardíaca são duas das mais temidas complicações do tratamento oncológico devido ao seu impacto no prognóstico cardiovascular e oncológico, podendo afetar ainda a escolha e manutenção das opções terapêuticas.

Diversas sociedades cardio-onco-hematológicas surgiram ao redor do mundo com o objetivo de gerar diretriz clínicas práticas e melhorar o diagnóstico e tratamento das complicações cardiovasculares resultantes das terapias oncológicas. A cardio-oncologia é uma disciplina em contínuo crescimento e desenvolvimento. Nós acreditamos fortemente que educação médica continuada e uma abordagem multidisciplinar são necessárias para um cuidado médico de qualidade. 
Este texto é o resultado de um trabalho multidisciplinar envolvendo cardiologistas, hematologistas e oncologistas. Nosso objetivo é de oferecer informação à equipe de cuidados em saúde envolvido na assistência destes pacientes. Devido à sua extensão, este texto será dividido em três partes.

Palavras-chave: QUIMIOTERAPIA

CÂNCER

CARDIOTOXICIDADE

CARDIO-ONCOLOGIA

DISFUNÇÃO VENTRICULAR

INSUFICIÊNCIA CARDÍACA

DETECÇÃO PRECOCE

SEGUIMENTO

\section{Isquemia miocárdica y enfermedad arterial coronaria Dras. Carolina Artucio ${ }^{4}$, Mónica Delorenzi ${ }^{5}$, Mariela Lujambio ${ }^{5}$}

Los tratamientos que incluyen quimioterapia, radioterapia y terapias hormonales exponen a los pacientes con cáncer a una amplia variedad de complicaciones cardiovasculares, dentro de las cuales se encuentra el desarrollo de enfermedad arterial coronaria (EAC).

El mecanismo por el cual se produce esta injuria es complejo y multifactorial, e incluye: daño endotelial directo, vasoespasmo, trombosis y liberación de factores citotóxicos. Se plantea que el cáncer y la enfermedad cardiovascular comparten vías fisiopatológicas en común ${ }^{(13)}$. Además, se producen modificaciones del metabolismo lipídico que llevan a aterogénesis precoz y/o rápida progresión de enfermedad coronaria preexistente $^{(4)}$.

Varios tratamientos para el cáncer inducen toxicidad vascular, de los cuales los más estudiados son la capecitabina y el 5-fluorouracilo (5-FU). Pueden producir isquemia silente o sintomática, en reposo o demostrable con test de inducción de isquemia, y puede cesar una vez suspendido o finalizado el tratamiento. En los casos más severos, pueden producir eventos mayores, muerte súbita o infarto de miocardio, siendo la manifestación más frecuente el síndrome coronario agudo sin elevación del segmento ST (SCAsST) ${ }^{(4)}$.

Las drogas más asociadas a la isquemia coronaria son los antimetabolitos, anticuerpos monoclonales inhibidores de Her2 y los inhibidores de diferentes moléculas (factor de crecimiento endotelial, inhibidores TK de moléculas pequeñas, protesoma, angiogénesis y agentes antimicrotúbulos) (tabla 1). La incidencia de infarto e isquemia reportada varían entre 7,6 a $63 \%$, dependiendo del tipo de tratamiento, dosis y modo de administración (bolo o infusión continua) $)^{(1,4,5)}$.

La radioterapia torácica, como monoterapia o asociada a quimioterapia, produce EAC macro y microangiopática con inflamación endotelial que acelera la aterogénesis y el riesgo de desarrollar IC, generalmente con fracción de eyección preservada. El riesgo de desarrollar EAC se incrementa a mayor dosis recibida, tiempo de evolución más prolongado y coexistencia de factores de riesgo. Tiene distribución típica, agrupada en sectores proximales de los vasos coronarios, lo que conlleva un riesgo de infarto extenso ${ }^{(7)}$. El daño vascular puede estar presente en áreas distantes al campo de radiación, cuando los pacientes reciben quimioterapia adicionada a la radioterapia ${ }^{(1,4)}$.

A su vez, los pacientes oncológicos presentan un estado protrombótico que promueve la aparición de síndromes coronarios agudos y la presencia de trombocitopenia no constituye un factor protector $^{(8-10)}$.

\section{Evaluación inicial y seguimiento}

Los pacientes deben ser informados del riesgo aumentado de desarrollar EAC al iniciar el tratamiento oncológico, y se deben aconsejar modificaciones apropiadas al estilo de vida como una dieta cardiosaludable, un plan de ejercicio personalizado, mantenimiento de un peso adecuado, control de factores de riesgo, incluyendo perfil lipídico con LDL objetivo < de $70 \mathrm{mg} / \mathrm{dL}$ en pacientes de alto riesgo o con EAC conocida y de $100 \mathrm{mg} / \mathrm{dL}$ en el resto de la población. Además, se debe explicar a los pacientes cuáles son los síntomas sugestivos

4. Instituto de Cardiología Intervencionista, INCI, Sanatorio Casa de Galicia, Montevideo, Uruguay.

5. Servicio Cardiología, Unidad de Cardio Oncología, Hospital Maciel, ASSE, Montevideo, Uruguay 


\begin{tabular}{|c|c|c|c|}
\hline $\begin{array}{l}\text { Agente } \\
\text { quimioterápico }\end{array}$ & Incidencia (\%) & Efectos cardiovasculares adversos & Mecanismo probable \\
\hline 5-Fluorouracilo & $0,1-19$ & Angina, vasoespasmo, infarto. & Vasoespasmo. \\
\hline Capecitabine & $0,02-10$ & Angina, vasoespasmo, infarto. & Vasoespasmo. \\
\hline Paclitaxel & $0,2-4$ & Angina, vasoespasmo, infarto. & Vasoespasmo. \\
\hline Bevacizumab & $1-6$ & Angina, infarto. & Injuria endotelial. \\
\hline Sunitinib & $1-13$ & $\begin{array}{l}\text { Angina, vasoespasmo, infarto, progresión } \\
\text { de enfermedad arterial coronaria. }\end{array}$ & Vasoespasmo. \\
\hline Radioterapia & $7,4-20$ & $\begin{array}{l}\text { Progresión de enfermedad arterial } \\
\text { coronaria. } \\
\text { Enfermedad vascular periférica. }\end{array}$ & $\begin{array}{l}\text { Injuria endotelial. } \\
\text { Rotura de placa. } \\
\text { Trombosis. }\end{array}$ \\
\hline
\end{tabular}

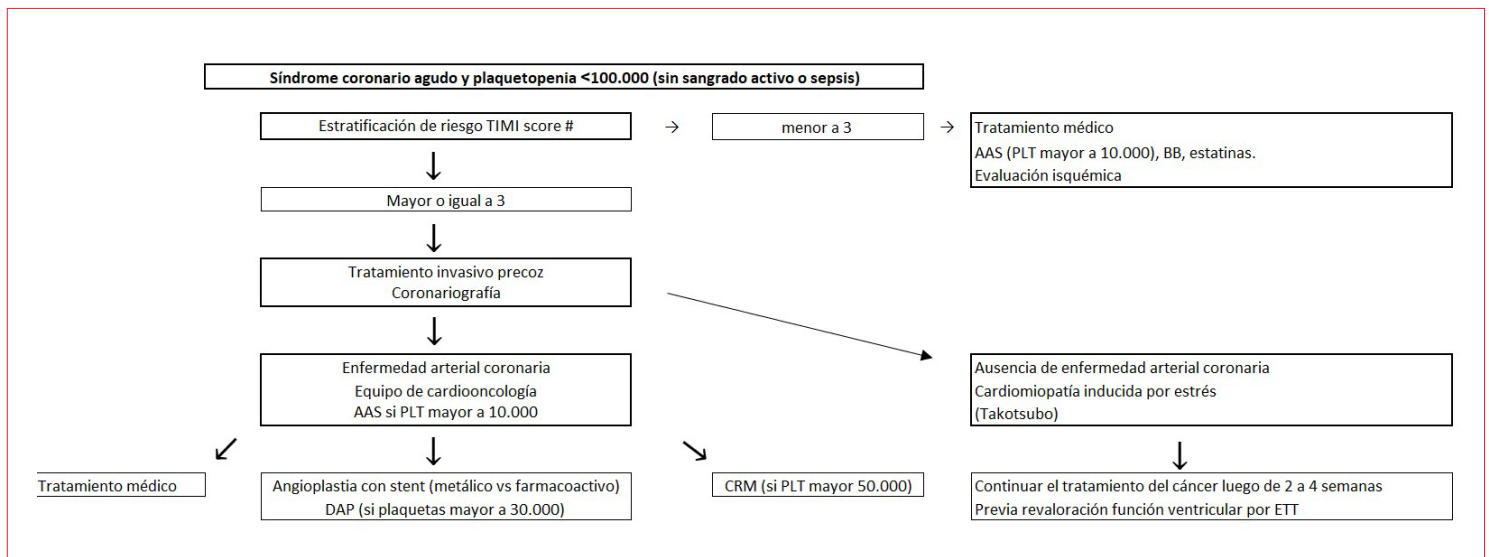

Figura 1. Estrategias de tratamiento en pacientes con síndrome coronario agudo, cáncer y plaquetopenia. Modificado de referencia 9.

\# Score TIMI para angina inestable o SCAcST más punto adicional para:

- antecedente de radiación al tórax (1 punto).

- tratamiento actual con quimioterapia protrombótica o Fase 1 (1 punto).

DAP: doble antiagregación plaquetaria; PLT: plaquetas; CRM: cirugía de revascularización miocárdica.

de afectación coronaria para que consulten de manera precoz ${ }^{(4)}$. En todos se recomienda un seguimiento anual con evaluaciones clínicas y electrocardiográficas ${ }^{(1,6)}$.

Diversos estudios sugieren que la EAC es silente en un gran porcentaje de pacientes con cáncer que recibieron radioterapia mediastinal. Por lo tanto, aún en pacientes asintomáticos, se recomienda controles a partir de los 5 años postratamiento y posteriores, por lo menos con estudios de provocación de isquemia miocárdica ${ }^{(1,4,6)}$.

\section{Diagnóstico y tratamiento}

El abordaje para el manejo de los pacientes que recibirán tratamientos que aceleran el proceso aterogénico con riesgo de desencadenar un SCA debe ser multidisciplinario. Es primordial identificar aquellos pacientes de alto riesgo, ya sea por ser portadores de EAC demostrada o por la pre- sencia de múltiples factores de riesgo. Especial atención merecen los pacientes con trombocitopenia, en quienes la antiagregación y la anticoagulación no siempre es posible $e^{(4,11)}$.

Frente al diagnóstico de un síndrome coronario agudo con elevación del segmento ST (SCAcST) o sin elevación del segmento ST (SCAsST) de alto riesgo, los pacientes con cáncer deben ser tratados de acuerdo con las recomendaciones vigentes para la población general. Según un registro multicéntrico, los pacientes con cáncer que presentan un SCAcST tuvieron mayor mortalidad que aquellos sin antecedentes oncológicos. En particular, el diagnóstico de cáncer realizado en los 6 meses previos al SCAcST es un predictor de mayor mortalidad ajustada a 7 días $^{(12)}$.

Para los pacientes con múltiples factores de riesgo o EAC previa, el diagnóstico y tratamiento farmacológico no difiere del paciente no oncológico (antiagregantes, estatinas, BB e IECA) ${ }^{(4,11)}$. La 
cirugía de revascularización cardíaca puede considerarse cuando el neoplasma es potencialmente curable o cuando el pronóstico estimado es aceptable. Si la sobrevida es menor a 1 año, solo se deben tratar los pacientes con SCA de alto riesgo o SCAcST. Para la angina inestable, se plantea iniciar tratamiento farmacológico y reservar la revascularización para algunas situaciones de riesgo mayor como la angina refractaria (figura 1$)^{(9)}$.

En el laboratorio de hemodinamia, pueden presentarse problemas únicos en la evaluación y tratamiento de los pacientes con cáncer ${ }^{(1,9,10)}$.

- La coronariografía diagnóstica puede ser realizada con cualquier conteo plaquetario.

- El AAS puede ser administrado si el recuento plaquetario es mayor a 10.000 por $\mathrm{mm}^{3}$.

- La doble antiagregación con AAS y clopidogrel puede ser administrada si el recuento plaquetario es de 30.000 a 50.000 por $\mathrm{mm}^{3}$.

- Prasugrel, ticagrelor o los inhibidores de la glucoproteína IIb/IIIa pueden administrarse si el recuento plaquetario es $>50.000$ por $\mathrm{mm}^{3}$.

- No se recomienda la transfusión plaquetaria profiláctica previa a la coronariografía, excepto si está recomendado explícitamente por el equipo hematooncológico.

La plaquetopenia por quimioterapia es transitoria, con un período de recuperación previsible, y un bajo riesgo de hemorragia grave con recuentos plaquetarios $>10.000$ por $\mathrm{mm}^{3}$. Para los pacientes con síndromes mielodisplásicos y trombocitopenia, habitualmente crónica, el riesgo de hemorragia es mayor que en la plaquetopenia inducida por quimioterapia.

$\mathrm{Al}$ realizar la angioplastia coronaria, algunas estrategias pueden evitar una hemorragia significativa, como utilizar acceso radial, realizar heparina en dosis bajas (3050 U/kg de heparina no fraccionada) si el recuento plaquetario es $<50.000$ por $\mathrm{mm}^{3} \mathrm{y}$ utilizar medidas de protección gástrica de rutina ${ }^{(13)}$.

Por otra parte, se debe evaluar las características de la lesión coronaria y de la enfermedad oncológica, estadio y terapéutica propuesta, reconociendo al cáncer como un estado protrombótico y proinflamatorio que suele asociarse con un mayor riesgo de trombosis intrastent y posiblemente reestenosis. A su vez, la quimioterapia retarda el proceso de reendotelización de los stents, por lo que puede ser necesario prolongar la doble antiagregación plaquetaria (DAP). También se debe re- conocer el mayor riesgo trombogénico de algunos fármacos, como el cisplatino y talidomida.

Con estas bases patogénicas se sugiere realizar angioplastia del vaso que tenga isquemia documentada, asegurarse el óptimo implante del stent con adecuada expansión, aposición, ausencia de disección de los bordes y, si es posible, realizar control mediante imágenes intravasculares posintervención. Se sugiere evitar la angioplastia de lesiones topografiadas en bifurcaciones, así como el implante de stents superpuestos, debido a que aumentan el riesgo de trombosis intrastent. Finalmente, se debe tener en cuenta que en algunas ocasiones será necesario la interrupción anticipada de la DAP.

En las situaciones en las que se realiza revascularización pueden considerarse las siguientes recomendaciones ${ }^{(9,10)}$ :

- La angioplastia con balón debería ser considerada para pacientes con cáncer que no sean candidatos para recibir DAP (recuento de plaquetas 10.00030 .000 por $\mathrm{mm}^{2}$ ) o cuando se planea un procedimiento o cirugía no cardíaca en un plazo menor a 4 semanas.

- Los stents metálicos que requieren DAP por un corto plazo deben considerarse en los pacientes con recuentos de plaquetas $>30.000$ por $\mathrm{mm}^{2}$ y aquellos que requieran un procedimiento no cardíaco, cirugía, o quimioterapia que puede posponerse más de 4 semanas.

- En los pacientes en los que no se plantea ningún procedimiento adicional, se pueden utilizar stents liberadores de fármacos de nueva generación.

- Cuando es necesario realizar cirugía no cardíaca de urgencia luego de la colocación de stent, se debe continuar con al menos un agente antiplaquetario.

- Si el recuento de plaquetas es $<50.000$ por $\mathrm{mm}^{3}$, la duración de la DAP puede limitarse a 2 semanas después de la ATC con balón, 4 semanas después del stent metálico y 6 meses tras implante de stents liberadores de fármacos de nueva generación.

Destacamos la importancia del trabajo multidisciplinario en la toma de decisiones. El equipo cardiológico no debe olvidar el corolario general según el cual el tratamiento de la enfermedad coronaria no debería retrasar el tratamiento oncológico potencialmente curativo. 


\section{Hipertensión arterial \\ Dras. Natalia Moreira ${ }^{6}$, Andrea Simeone ${ }^{7}$}

La hipertensión arterial (HTA) es la comorbilidad más frecuente en los pacientes con cáncer ${ }^{(14)}$. Su diagnóstico y tratamiento adecuado resulta fundamental, pues constituye un factor de riesgo para el desarrollo de IC vinculada a cardiotoxicidad $^{(4,15)}$. El antecedente de HTA en pacientes que reciben tratamiento con antracíclicos implica un incremento del riesgo de IC congestiva de 1,58 veces y el desarrollo de HTA ha sido propuesto como uno de los mecanismos causales de disfunción ventricular izquierda en pacientes que reciben agentes angiogénicos ${ }^{(16,17)}$.

La incidencia de HTA varía con el tipo de tumor; es menor en el melanoma, tumores de partes blandas y cáncer de mama, teniendo mayor incidencia en el cáncer renal, pulmonar, de ovario, de próstata, colorrectal y gástrico ${ }^{(18,19)}$.

\section{Inhibidores de la angiogénesis}

Varias drogas utilizadas en el tratamiento del cáncer conllevan el desarrollo de HTA como efecto adverso. Dentro de estas se destacan los inhibidores de la angiogénesis como el bevacizumab, que interactúan con el factor de crecimiento endotelial vascular (VEGF), pudiendo contribuir al desarrollo de HTA de novo o agravar la preexistente con una frecuencia que varía entre el $11 \%$ y $43 \%$ en distintos estudios. Esta incidencia varía en relación con el tipo de tumor y la coadministración de otras drogas ${ }^{(15,20)}$.

Como mecanismos vinculados a la HTA en este contexto, se reconocen la interferencia con la vasodilatación a través de la disminución de óxido nítrico y el aumento de endotelina, así como también activación de otros sistemas neurohumorales, rarefacción vascular (disminución de la densidad arteriolar y capilar) y daño renal glomerular con distintos grados de proteinuria ${ }^{(21)}$. La HTA suele desarrollarse al inicio del tratamiento, pudiendo presentarse hasta un año tras iniciar este ${ }^{(4,22)}$.

Existen dos tipos de fármacos que interactúan con el VEGF:

- Anticuerpos monoclonales dirigidos contra el VEGF, dentro de los que se destaca el bevacizumab, cuyo efecto prohipertensivo es dosis dependiente y prolongado, ya que tiene una vida media cercana a los 20 días ${ }^{(23)}$.

- Inhibidores de la tirosina quinasa que interfieren en la unión del VEGF con su receptor. Se incluyen aquí sunitinib, axitinib, sorafenib, pazopanib, lenvatinib, vandetanib y regorafenib, que se utilizan habitualmente como monoterapia por períodos prolongados, lo que aumenta el tiempo de exposición al efecto prohipertensivo ${ }^{(19,24)}$.

Se ha demostrado que el desarrollo de HTA es un efecto de clase y puede ser considerado un marcador de eficacia terapéutica, con mejores resultados oncológicos y mayor sobrevida en los pacientes en que se manifiesta este efecto prohipertensivo, sin afectarse este efecto terapéutico con el control farmacológico de la HTA ${ }^{(25-28)}$.

\section{HTA y tratamientos concomitantes no oncoespecíficos}

Diversas drogas utilizadas en apoyo al paciente en tratamiento antineoplásico pueden contribuir o potenciar el efecto prohipertensivo inducido por la quimioterapia ${ }^{(29)}$. Los antiinflamatorios no esteroideos (AINE), la eritropoyetina y los corticoesteroides son los más frecuentes. También hay que evaluar la utilización de tratamientos hormonales, inmunosupresores, aminas simpaticomiméticas (descongestivos nasales, antigripales), psicofármacos antipsicóticos (clozapina) y antidepresivos (IMAO, tricíclicos y algunos ISRS). Por último, no debe olvidarse el consumo excesivo de alcohol, cafeína y drogas de abuso como la cocaína, que también inducen $\mathrm{HTA}^{(19)}$.

Los AINE son utilizados con frecuencia para lograr analgesia en los pacientes con cáncer. Estos inducen HTA al inhibir de forma no selectiva la ciclooxigenasa, lo que genera reducción de la síntesis de prostaglandinas y, consecuentemente, vasoconstricción renal y retención hidrosalina. Se ha estimado que el riesgo de desarrollar HTA se duplica en aquellos pacientes normotensos que reciben con regularidad un AINE, siendo este efecto más marcado a mayor edad o en presencia de función renal alterada ${ }^{(30)}$. El consumo de AINE puede descontrolar la HTA en pacientes tratados con IECA, ARA-II, BB y diuréticos, debido a 


\begin{tabular}{|c|c|c|}
\hline Clase de droga & Preferencia & Precauciones \\
\hline $\begin{array}{l}\text { Inhibidores de la enzima } \\
\text { convertidora de angiotensina } \\
\text { (IECA) }\end{array}$ & $\begin{array}{l}\text { Disfunción sistólica del ventrículo izquierdo. } \\
\text { Nefropatía diabética. }\end{array}$ & $\begin{array}{l}\text { Alteración de la función renal. } \\
\text { Hiperkalemia. }\end{array}$ \\
\hline $\begin{array}{l}\text { Antagonistas de } \\
\text { angiotensina II (ARA-II) }\end{array}$ & $\begin{array}{l}\text { Disfunción sistólica del ventrículo izquierdo } \\
\text { (intoleracia a IECA). } \\
\text { Nefropatía diabética. }\end{array}$ & $\begin{array}{l}\text { Alteración de la función renal. } \\
\text { Hiperkalemia. }\end{array}$ \\
\hline Betabloqueantes & $\begin{array}{l}\text { Disfunción sistólica del ventrículo izquierdo. } \\
\text { Cardiopatía isquémica. } \\
\text { Angor. }\end{array}$ & $\begin{array}{l}\text { Bradiarritmia. } \\
\text { Diabetes (hipoglicemia). }\end{array}$ \\
\hline $\begin{array}{l}\text { Calcioantagonistas } \\
\text { dihidropiridínicos }\end{array}$ & $\begin{array}{l}\text { Hipertensión sistólica aislada. } \\
\text { Ancianos. }\end{array}$ & $\begin{array}{l}\text { Edemas preexistentes de } \\
\text { miembros inferiores. }\end{array}$ \\
\hline Diuréticos tiazídicos & $\begin{array}{l}\text { Hipertensión arterial que requiere } \\
\text { combinación de múltiples fármacos. }\end{array}$ & $\begin{array}{l}\text { Hiperuricemia. } \\
\text { Hipokalemia. } \\
\text { Deshidratación }\end{array}$ \\
\hline
\end{tabular}

la importancia del efecto vasodilatador renal de las prostaglandinas en el mecanismo de acción de estos fármacos. Sin embargo, no se ha demostrado interferencia con agentes calcioantagonistas o bloqueadores adrenérgicos a nivel central ${ }^{(19)}$.

La eritropoyetina es una droga utilizada para la estimulación de la formación de glóbulos rojos en la médula ósea. Aproximadamente el 30\% de los pacientes que reciben tratamiento con eritropoyetina presentan aumento de las resistencias vasculares periféricas y algún grado de ascenso de la PA. Este efecto puede aparecer a las dos semanas de iniciado el tratamiento. La HTA no controlada puede obligar a la suspensión del tratamiento oncológico en un $15 \%$ de los casos; habitualmente responde bien al manejo del volumen $\mathrm{y}$ agentes antihipertensivos de cualquier clase ${ }^{(19)}$.

Los corticoesteroides tienen diversos usos en contextos de tratamiento oncológico. Debido a su acción mineralocorticoide, inducen retención hidrosalina e incrementan la síntesis de angiotensinógeno con el consecuente aumento de la PA. Entre los pacientes que reciben corticoides en regímenes prolongados, al menos un $20 \%$ presentan elevación significativa de las cifras tensionales, lo cual depende de la dosis indicada y es más marcado en pacientes de edad avanzada, HTA previa o función renal alterada. Este efecto prohipertensivo se contrarresta con agentes diuréticos e $\mathrm{IECA}^{(19,30)}$.

\section{Diagnóstico y manejo terapéutico}

El adecuado manejo de la HTA pretende disminuir el riesgo de complicaciones cardiovasculares a corto plazo y permitir el mantenimiento de una terapia antiangiogénica eficaz. Las guías internacionales recomiendan estratificar el riesgo $\mathrm{CV}$ previo al inicio de inhibidores antiVEGF, diagnosticar oportunamente la HTA, definida como una PA mayor o igual a $140 / 90 \mathrm{mmHg}$ y optimizar el tratamiento antihipertensivo en caso de HTA preexistente, de modo que la HTA esté controlada de manera adecuada ${ }^{(4,28,31,32)}$.

El aumento de cifras tensionales vinculado a la terapia antiangiogénica ocurre en los primeros meses del tratamiento, por lo que la PA debe controlarse semanalmente al inicio de este y luego cada dos a tres semanas. Al finalizar el primer ciclo, y si las cifras se mantienen estables, se recomienda el control tensional en cada consulta o mediante automonitoreo domiciliario, según las características del paciente ${ }^{(31,32)}$.

Se recomienda mantener una PA objetivo menor a 140/90 $\mathrm{mmHg}$ al igual que en la población general, o menor a 130/80 mmHg en casos seleccionados. Las guías de práctica clínica actuales sugieren iniciar o ajustar el tratamiento farmacológico antihipertensivo cuando los pacientes presentan PA mayor o igual a 140/90 mmHg o aumentos de la PA diastólica mayores o iguales a $20 \mathrm{mmHg}$ respecto a los valores previos al tratamiento $^{(31,32)}$.

El manejo de la HTA debe ser inmediato y agresivo, para evitar daño de órgano blanco y dificultades en el tratamiento oncológico. Se sugiere restringir la ingesta de sodio, siendo limitadas otras medidas no farmacológicas por condiciones nutricionales y vinculadas a la capacidad de realizar actividad física.

Distintos fármacos antihipertensivos de primera línea han demostrado ser efectivos para el control de la HTA inducida por antiangiogénicos. Para su elección se deben tener en cuenta las características del paciente y los posibles efectos 


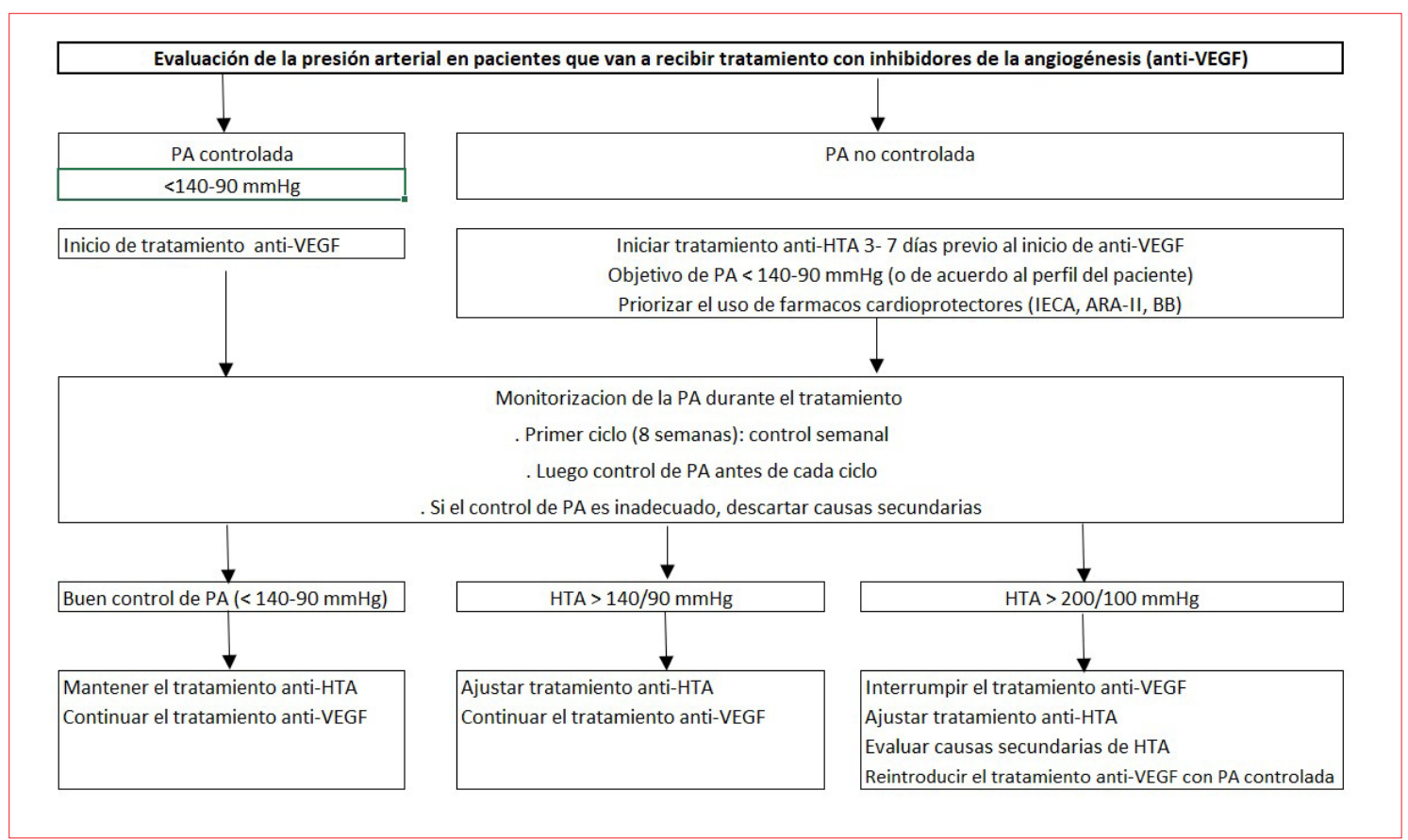

Figura 2. Evaluación de la presión arterial en pacientes que van a recibir tratamiento con inhibidores de la angiogénesis (antiVEGF). Modificado de referencia 28.

anti-VEGF: inhibidores del factor de crecimiento del endotelio vascular; IECA: inhibidores de la enzima convertidora de angiotensina; ARA-II: antagonistas de los receptores de la angiotensina II; BB: beta bloqueantes; PA: presión arterial; HTA: hipertensión arterial.

adversos de su uso (tabla 2) ${ }^{(19,31)}$. Caben aquí las siguientes apreciaciones:

- Los IECA, ARA-II y calcioantagonistas dihidropiridínicos (como la amlodipina) son considerados como fármacos de primera línea en opinión de expertos, quienes recomiendan la combinación de IECA o ARA-II con amlodipina como estrategia eficaz en la mayoría de los casos. Los IECA tienen la ventaja de lograr descensos más rápidos en la $\mathrm{PA}^{(31)}$.

- Se sugiere el uso IECA y BB en aquellos pacientes que presentan riesgo elevado de desarrollar insuficiencia cardíaca o disfunción ventricular izquierda ${ }^{(4)}$. El uso de nebivolol se considera una buena opción por su acción vasodilatadora dependiente del aumento de la disponibilidad de óxido nítrico ${ }^{(4,21,33)}$. También se considera razonable el uso de otros betabloqueantes con acción vasodilatadora como carvedilol $^{(4)}$.

- Los calcioantagonistas no dihidropiridínicos (diltiazem y verapamil) deben ser evitados por su efecto inhibitorio sobre la citocromo P450 3A4 que metaboliza algunos de los inhibidores de la angiogénesis, con el consiguiente aumento de los niveles plasmáticos de estos fármacos y mayor riesgo de toxicidad ${ }^{(4,21,22,31)}$.

- Se debe tener precaución con el uso de diuréti- cos, por el riesgo de alteraciones hidroelectrolíticas y prolongación del intervalo QT, ya que los antiVEGF pueden provocar diarrea y potencial deshidratación como efectos adversos ${ }^{(4,19,31)}$.

\section{Modificación del tratamiento oncológico}

El efecto antihipertensivo de los inhibidores de la angiogénesis (antiVEGF) es reversible, por lo que disminuir la dosis o interrumpir temporalmente su uso puede ser necesario como último recurso para lograr el control de las cifras de PA en caso de HTA severa (figura 2). Esto aplica fundamentalmente para los inhibidores de la tirosina quinasa, que son administrados por vía oral durante períodos prolongados, pudiendo reiniciarlos o ajustar su dosis una vez que la HTA se ha controlado. Cuando se administran agentes por vía intravenosa como bevacizumab, se recomienda no administrarlo si la PA sistólica es mayor de 160 mmHg o la PA diastólica es mayor de $100 \mathrm{mmHg}$. Una vez controladas la cifras tensionales durante el período de suspensión, habitualmente de cuatro semanas, la droga puede volver a administrarse ${ }^{(11)}$.

Hay que tener en cuenta que, una vez finalizado el tratamiento con estos agentes, es usual que la PA vuelva otra vez a sus niveles previos, por lo que se puede requerir reducir o eventualmente suspender el tratamiento antihipertensivo ${ }^{(19)}$. 


\section{Miocardiopatía por estrés y miocarditis por ICI Dres. Alexis Dini ${ }^{8}$, Andrés Gari ${ }^{8}$, Andreina Gómez ${ }^{1}$, Verónica Montauban ${ }^{8}$, Giovanna Podstavka ${ }^{8}$}

\section{Miocardiopatía por estrés secundario a quimioterápicos}

La miocardiopatía por stress o Síndrome de Takotsubo (ST) se define de acuerdo con el documento de consenso publicado en 2018 (InterTAK Diagnostic Criteria) como la presencia de disfunción transitoria del VI que habitualmente excede un territorio coronario, puede estar precedida de estrés emocional, físico o ambos (aunque no es excluyente), con síntomas similares a un SCA y frecuentemente asociado a alteraciones nuevas en el ECG y elevación moderada de biomarcadores $\operatorname{cardíacos}^{(34)}$.

La incidencia real de ST en pacientes con cáncer es desconocida, estimada en $1,13 \%$. Sin embargo, los pacientes con cáncer tienen un riesgo aumentado de ST y además el pronóstico es peor, con mayor mortalidad (13,8 vs. $2,9 \%$ ) comparados con pacientes sin patología oncológica ${ }^{(35)}$.

Los factores desencadenantes para el ST en pacientes con cáncer incluyen el estrés emocional y psicosocial, el estado inflamatorio inducido por el cáncer y el potencial estrés físico asociado a la cirugía, la radioterapia y la quimioterapia sistémica. Existe además la hipótesis de que ciertos mediadores paraneoplásicos circulantes podrían modificar los receptores adrenérgicos en el tejido cardíaco, predisponiendo al desarrollo de disfunción contráctil ${ }^{(36)}$.

Varias drogas quimioterápicas se han asociado con el desarrollo de ST. El fármaco más frecuentemente vinculado al desarrollo de ST ha sido el 5FU, si bien la principal manifestación de cardiotoxicidad de las flouropirimidinas está vinculada a la afectación coronaria ${ }^{(37)}$. El 5FU puede ser administrado por vía intravenosa en bolo o infusión, para el tratamiento de tumores sólidos, incluyendo cáncer colorrectal, gástrico, esofágico, pancreático, de próstata, de vejiga y de mama. En la mayoría de los casos, el ST ocurre durante o inmediatamente posterior a la infusión y durante el primer ciclo de 5FU. La reintroducción de 5FU es motivo de controversia, con reportes que muestran una mortalidad cercana al $13 \%$ con la reintroducción ${ }^{(38)}$. El capecitabine es una flouropirimidina de administración oral que se transforma en 5FU por acción enzimática en los tejidos tumorales, y tiene efectos cardiotóxicos similares al $5 \mathrm{FU}^{(39)}$. Otras drogas que pueden estar implicadas incluyen bevacizumab, rituximab, inhibidores de VEGF e inhibidores de angiogénesis ${ }^{(40,41)}$.

En un paciente que se presenta con dolor torácico o disnea durante o luego del tratamiento con quimioterapia, el diagnóstico diferencial del SCA debe incluir la tromboembolia venosa, IC, miocarditis y ST. La presencia de nuevas alteraciones en la motilidad parietal en segmentos medioapicales que exceden un territorio coronario, en ausencia de accidente de placa de ateroma, apoyan el planteo de ST. A pesar de que el pronóstico del ST es en general bueno, la interrupción del tratamiento quimioterápico puede afectar de forma adversa el pronóstico oncológico ${ }^{(42,43)}$.

\section{Miocarditis por inhibidores de checkpoint inmunológico}

La miocarditis, definida como una enfermedad inflamatoria del músculo cardíaco, es una causa importante de insuficiencia cardíaca aguda, muerte súbita y miocardiopatía dilatada. Puede ser causada por infecciones (principalmente virus), fármacos, sustancias tóxicas o trastornos autoinmunitarios. Durante la fase aguda, un desencadenante específico induce una respuesta inmunitaria que puede ser de diferente gravedad, desde transitoria y leve hasta fulminante ${ }^{(44)}$. Histológicamente se define como la presencia de infiltrados inflamatorios en el miocardio, asociados a una degeneración miocitaria y necrosis de causa no isquémica, de acuerdo con los criterios histológicos de Dallas $^{(45)}$.

La resonancia magnética cardíaca (RMC) provee una evaluación no invasiva muy útil para confirmar el diagnóstico de miocarditis, sobre todo en la fase aguda de la enfermedad. Los criterios de Lake Louise incluyen: a) aumento de la señal focal o difusa en las secuencias potenciadas en $\mathrm{T} 2$; b) realce precoz con gadolinio, midiendo el realce absoluto total o el realce total relati- 
vo del miocardio; y c) al menos un foco de realce tardío focal no isquémico ${ }^{(46)}$. Cuando se cumplen al menos dos de dichos criterios, se obtiene una sensibilidad del 76\% y una especificidad del 96\% en pacientes con sospecha clínica de miocarditis aguda ${ }^{(47)}$. Además, estudios recientes han demostrado que existe una buena correlación entre los resultados de la RMC y la biopsia endomiocárdica en la miocarditis aguda, con una concordancia de hasta el 79\% al utilizar las nuevas técnicas de $\mathrm{RMC}^{(48)}$.

Los inhibidores de checkpoint inmunológico (ICI) son anticuerpos monoclonales dirigidos contra moléculas del checkpoint inmunológico CTLA4 (ipilimumab), PD1 (nivolumab y pembrolizumab) y PDL1 (atezolizumab, avelumab, durvalumab). $\mathrm{Al}$ combinar diferentes ICI, se bloquean distintas señales inmunológicas inhibitorias del microambiente tumoral. Son utilizados en el tratamiento de tumores sólidos (melanoma, cáncer pulmonar de células no pequeñas, cáncer de células renales, cáncer de ovario) y hematológicos (linfoma de Hodgkin, linfoma primario, leucemia linfoblástica crónica y mieloma múltiple) ${ }^{(49-51)}$.

Estos agentes pueden producir un espectro amplio de eventos adversos inmunorelacionados, con afectación neurológica, endócrina, pulmonar, gastrointestinal, renal y cardíaca. Afectan hasta el 90\% de los pacientes, pero las manifestaciones graves son raras ${ }^{(52-55)}$.

Los ICI reactivan la respuesta inmunitaria antitumoral con activación de células T contra antígenos comunes al tumor y a los cardiomiocitos (expresión constitutiva de PD1 y PD-L1) ${ }^{(56)}$, generando injuria miocárdica directa, ruptura de la membrana del miocito (con elevación de troponina $\mathrm{T}$ e I) e infiltración linfocitaria (a predominio de linfocitos T citotóxicos) $)^{(57-59)}$.

La incidencia de miocarditis por ICI se encuentra en torno al 0,09-1,1\%, y aumenta con tratamiento combinado a $0,27-2,4 \%{ }^{(59,60)}$. El diagnóstico de miocarditis se realiza más frecuentemente en torno a 30 días de iniciado el tratamiento y más del 90\% tienen elevación de troponinas. Si bien la incidencia de miocarditis por ICI es baja, hasta en un $46 \%$ manifiesta efectos adversos CV mayores como muerte, shock cardiogénico, arritmias ven- triculares y BAVC. En estos pacientes, la elevación de las troponinas séricas suele ser mayor ${ }^{(61)}$.

Aproximadamente el 50\% de los pacientes que desarrollan miocarditis por ICI no experimentaron otro efecto adverso, pero pueden tener antecedentes de hepatitis o, de manera simultánea, miositis con rabdomiólisis ${ }^{(60-62)}$. A su vez, los pacientes con desórdenes autoinmunes sistémicos pueden tener miocarditis subclínica ${ }^{(59)}$.

Dado que la toxicidad suele ocurrir a las 2 o 3 semanas de iniciado el tratamiento (y aún con ECG y ETT basales normales), se recomienda la dosificación seriada de troponinas en situación basal y luego de cada ciclo. Su aumento amerita una evaluación más exhaustiva ${ }^{(63)}$. En este contexto clínico, la RMC se torna necesaria para confirmar el diagnóstico ${ }^{(28)}$.

El tratamiento inicial implica la suspensión inmediata de ICI y la administración de corticoides a dosis altas (metilprednisolona) ${ }^{(61)}$ ya que estos han demostrado mejorar la fracción de eyección de ventrículo izquierdo (FEVI) en el 50\% de los pacientes $^{(61,63)}$. Las directrices de la American Society for Clinical Oncology recomiendan el uso de prednisona o metilprednisolona en dosis de 1 o $2 \mathrm{mg} / \mathrm{Kg}$ día. Para casos que no respondan precozmente a las dosis altas de corticoides, se pueden utilizar dosis mayores de metilprednisolona (1 g/día IV) y/o asociar micofenolato mofetilo, infliximab o globulinas antitimocito $^{(64)}$. Los pacientes que desarrollan deterioro de la FEVI deberían ser tratados de acuerdo con las recomendaciones internacionales para el tratamiento de la $\mathrm{IC}^{(64,65)}$.

La miocarditis por ICI es una complicación grave y poco frecuente, que debe confirmarse por RMC. El manejo sigue los mismos criterios que en la población general ${ }^{(66)}$. Sin embargo, los pacientes con cáncer tienen opciones terapéuticas limitadas para el manejo de la IC avanzada. Además, la presencia de una neoplasia activa es una contraindicación absoluta para el trasplante cardíaco y dificulta la consideración de asistencia circulatoria mecánica ventricular de larga duración ${ }^{(61)}$. La detección y el tratamiento precoces previenen la presentación fulminante de la miocarditis, la secuela tardía de fibrosis y el desarrollo de miocardiopatía dilatada ${ }^{(67)}$. 


\section{Alteraciones del ritmo cardíaco \\ Dras. Valentina Agorrody ${ }^{9}$, Soledad Murguía ${ }^{10}$, Andrea Simeone ${ }^{9}$}

Diferentes alteraciones del ritmo y trastornos de la conducción cardíaca se pueden generar durante el tratamiento con quimioterápicos. Puede tratarse de arritmias benignas o complejas, pudiendo determinar, en ocasiones, la discontinuación o suspensión del tratamiento oncoespecífico. En la mayoría de los pacientes, las arritmias se asocian con cambios metabólicos y del medio interno; en estos casos, se trata de eventos transitorios que se suelen revertir con el tratamiento ${ }^{(68)}$.

Por desgracia, no se disponen datos claros ni contundentes sobre la incidencia real de las diferentes arritmias y su asociación evolutiva con el tratamiento oncológico, aunque es probable que esté subestimada, debida a la ausencia de una sistematización en su registro y la frecuente exclusión de pacientes con riesgo $\mathrm{CV}$ elevado en los grandes estudios observacionales.

La arritmia sostenida más frecuente en el paciente oncológico es la fibrilación auricular (FA). En términos generales, el diagnóstico de FA en este contexto clínico obliga a instaurar una estrategia de control de frecuencia en paralelo con la estratificación del riesgo tromboembólico versus hemorrágico, definiendo la indicación de anticoagulación, siguiendo las pautas correspondientes al manejo de la FA no valvular en pacientes con cáncer.

En la tabla 3 se detallan las diferentes arritmias relacionadas con las drogas utilizadas en el tratamiento del cáncer ${ }^{(11)}$. Nunca debe olvidarse una regla de buena práctica clínica, según la cual siempre debe obtenerse un ECG basal y trazados adicionales durante el seguimiento en todo paciente que vaya a recibir fármacos potencialmente arritmogénicos.

\section{Fibrilación auricular y cáncer}

Está claro que los pacientes con cáncer tienen una mayor incidencia de FA. La presencia de FA incrementa el riesgo de fenómenos tromboembólicos e IC, aún en pacientes sin disfunción sistólica inducida por quimoterápicos. Un metaanálisis recientemente publicado por Yuan y col. ${ }^{(69)}$ demostró que esta población tiene hasta $47 \%$ más riesgo de desarrollar FA comparado con los pacientes sin cáncer. Sin embargo, es difícil distinguir causali- dad asociada a la quimioterapia, debido al interjuego de múltiples factores asociados a la FA como la edad, el tipo de cáncer, la modalidad de cirugía realizada y los diferentes regímenes de agentes quimioterápicos ${ }^{(70)}$. En cuanto al tipo de cáncer, se ha reportado una fuerte asociación de la FA con el mieloma múltiple y el cáncer de esófago ${ }^{(71)}$. En este estudio, el riesgo de FA fue mayor en los 90 días posteriores al diagnóstico, lo cual podría estar vinculado a los controles médicos más frecuentes, aunque también al inicio del tratamiento. A su vez, en los períodos intra o posoperatorio se pueden generar diferentes condiciones de disbalance autonómico, estrés, inflamación sistémica, trastornos hidroelectrolíticos o isquemia miocárdica, los cuales pueden gatillar uno o más episodios de FA. Esto se observa en particular durante el posoperatorio de la cirugía de tumores intratorácicos.

Por otro lado, el inicio de la quimioterapia sistémica también incrementa el riesgo de FA, en especial en determinados escenarios clínicos y en pacientes sometidos a algunos regímenes terapéuticos.

Es bien conocido el efecto deletéreo de la doxorrubicina y otras antraciclinas sobre la función sistólica del ventrículo izquierdo. Así, por ejemplo, un estudio publicado por Kilickap y col. demostró que, luego de la primera infusión de doxorrubicina, el riesgo de FA paroxística ya ascendía a 10,3\% en una monitorización de 48 horas $^{(72)}$. Aunque la frecuencia con que se desarrolla disfunción del VI secundaria a estos fármacos es considerable, no es mayor a la observada en pacientes con miocardiopatías dilatadas no isquémicas de otro origen ${ }^{(73)}$.

En pacientes hematooncológicos que reciben trasplante de precursores hematopoyéticos, se ha observado una incidencia de FA de hasta 27\%; un riesgo que es mayor aún en aquellos que reciben melfalán como régimen de precondicionamiento ${ }^{(74,75)}$

Una elevada proporción de pacientes con mieloma múltiple desarrollan FA. Esto podría explicarse, al menos en parte, por el uso de inhibidores del proteasoma, como carfilzomib. La incidencia de FA en quienes reciben este fármaco es incluso mayor que aquellos sometidos a terapia con otros inhibidores del proteasoma ${ }^{(76)}$. 


\begin{tabular}{|c|c|}
\hline Arritmias & Agentes quimioterápicos asociados a su desarrollo \\
\hline Bradicardia & $\begin{array}{l}\text { Tripóxido de arsénico, bortezomib, capecitabina, cisplatino, ciclofosfamida, } \\
\text { doxorrubicina, epirubicina, } 5 \mathrm{FU} \text {, ifosfamida, IL2, mitoxantrona, paclitaxel, } \\
\text { rituximab, talidomida. }\end{array}$ \\
\hline QT prolongado & $\begin{array}{l}\text { Doxorrubicina, epirrubicina, tamoxifeno, imatinib, sunitinib, sorafenib, nilotinib, } \\
\text { dasatinib, lapatinib, } 5 \mathrm{FU} \text {, bortezomib. }\end{array}$ \\
\hline Taquicardia sinusal & Antraciclinas, carmustine. \\
\hline $\begin{array}{l}\text { Bloqueo auriculo- } \\
\text { ventricular }\end{array}$ & $\begin{array}{l}\text { Antraciclinas, trióxido de arsénico, bortezomib, ciclofosfamida, } 5 \mathrm{FU} \text {, mitoxantrona, } \\
\text { rituximab, taxanos, talidomida. }\end{array}$ \\
\hline Fibrilación auricular & $\begin{array}{l}\text { Alquilantes (cisplatino, ciclofofamida, ifosfamida, melphalan), antraciclinas, } \\
\text { antimetabolitos (capecitabine, 5FU, gemcitabina), IL2, interferones, rituximab, } \\
\text { sorafenib, sunitinib, ibrutinib, inhibidores topoisomerasa II (amsacrina, etopósido), } \\
\text { taxanos, alcaloides de la vinca. }\end{array}$ \\
\hline $\begin{array}{l}\text { Taquicardia } \\
\text { supraventricular }\end{array}$ & $\begin{array}{l}\text { Alquilantes (cisplatino, ciclofofamida, ifosfamida), antraciclinas, antimetabolitos } \\
\text { (capecitabina, 5FU, metrotexate), bortezomib, doxorubicina, IL2, interferones, } \\
\text { paclitaxel. }\end{array}$ \\
\hline $\begin{array}{l}\text { Taquicardia/ } \\
\text { fibrilación ventricular }\end{array}$ & $\begin{array}{l}\text { Alquilantes (cisplatino, ciclofofamida, ifosfamida), antimetabolitos (capecitabine, } \\
\text { 5FU, gemcitabina), arsénico, doxorubicina, interferones, IL2, metrotexate, paclitaxel, } \\
\text { inhibidores de la proteasoma (bortezomib, carfilzomib), rituximab. }\end{array}$ \\
\hline Muerte súbita & $\begin{array}{l}\text { Trióxido de arsénico (torsade de pointes), } 5 \mathrm{FU} \text { (vasoespasmo e isquemia miocárdica), } \\
\text { interferones, nilotinib. }\end{array}$ \\
\hline
\end{tabular}

Los inhibidores de la tirosina quinasa actúan en varias vías de señalización del crecimiento celular. El factor de crecimiento vascular endotelial se ha reconocido como una causa de HTA, FEVI baja y, además, $\mathrm{FA}^{(77,78)}$.

El ibrutinib es un inhibidor de la tirosina quinasa de Bruton que ha revolucionado el tratamiento de varias linfopatías, entre ellas la LLC. Este fármaco merece mención especial por la fuerte asociación con FA y por los problemas relacionados con su manejo. En un metaanálisis reciente se ha reportado un riesgo relativo de FA de 8,81 en pacientes que reciben este tratamiento, comparado con agentes alternativos o placebo. Este riesgo aumenta con el tiempo de uso y la dosis ${ }^{(79)}$. Además, ibrutinib se metaboliza por la citocromo $\mathrm{P} 450$ y es un inhibidor de la glicoproteína $\mathrm{P}$, lo que predispone a interacciones farmacológicas con diversas drogas de amplio uso para control de frecuencia, reversión a ritmo sinusal y prevención de embolias sistémicas como verapamil, diltiazem, amiodarona, digoxina y agentes anticoagulantes directos.

\section{Manejo de la FA en los pacientes con cáncer}

A grandes rasgos, el manejo terapéutico de la FA en la población de pacientes con cáncer es el mismo que para los pacientes que no reciben quimioterapia. Sin embargo, en el paciente oncológico debe prestarse especial atención a las po- sibles y múltiples interacciones farmacológicas entre antiarrítmicos y quimioterápicos. Como muchos agentes antineoplásicos se metabolizan por vías comunes con diversos antiarrítmicos de uso frecuente, su efecto se podría ver potenciado. Es recomendable consultar tablas para evaluar la necesidad de reducir las dosis usuales previo al inicio de su administración y durante el seguimiento clínico ${ }^{(80)}$.

En particular, los agentes calcioantagonistas y la amiodarona interactúan con la mayoría de los citostáticos, por lo que deben utilizarse en dosis bajas. Por el contrario, las drogas antiarrítmicas de clase IA o IC suelen producir diversos grados de prolongación del intervalo QT. Dentro de la familia de los fármacos betabloqueantes, se describen mayores interacciones de carvedilol y propranolol con los quimioterápicos. Por lo tanto, durante el tratamiento oncológico se torna necesario monitorizar con frecuencia la aparición de bradicardia, la prolongación del intervalo QT y la aparición de arritmias ventriculares.

\section{Manejo del riesgo tromboembólico e indicación de anticoagulación oral en la FA del paciente oncológico}

La tradicional estratificación del riesgo tromboembólico que se requiere en los pacientes con FA puede no ser aplicable en los pacientes con 
cáncer. En un estudio previo, un score $\mathrm{CHADS}_{2}$ elevado se asoció con un mayor riesgo en pacientes con FA previa y cáncer, pero no en los que la desarrollaron luego del diagnóstico(81). Además, el score CHADS-VASC aún no se ha validado en este grupo de pacientes. Sin embargo, la decisión de iniciar la terapia con anticoagulación oral puede ser muy desafiante, debido a la coexistencia de varios factores de riesgo tromboembólicos clásicos no oncológicos, diversas interacciones farmacológicas con los anticoagulantes, predisposición a sangrados inherente al terreno y un riesgo tromboembólico elevado en relación con el propio cáncer.

A la fecha no se han publicado guías terapéuticas específicas para la elección de la terapia anticoagulante más adecuada en los pacientes con cáncer. La terapia con warfarina, de bajo costo y gran accesibilidad, acarrea varios problemas vinculados a la dificultad de lograr un adecuado tiempo en rango terapéutico por sus interacciones hepáticas, modificaciones en la dieta, suspensiones previas a procedimientos diagnósticos invasivos o cirugías, etc. Por otro lado, el uso de las heparinas de bajo peso molecular está bien establecido para la prevención de la enfermedad tromboembólica venosa, pero no para la terapia sistemática a mediano ni largo plazo en los pacientes con FA y cáncer.

La falta de evidencias contundentes sobre la terapia óptima surge principalmente de que los pacientes con cáncer han sido excluidos o representan una baja proporción en los grandes estudios que evaluaron la efectividad de los agentes anticoagulantes directos. Aunque algunos subanálisis de los ensayos ARISTOTLE y ENGAGE $\mathrm{AF}$ han sido alentadores en este sentido, estos datos precisan ser sometidos al escrutinio de investigaciones específicas. Como si fuera poco, las fluctuaciones en la función hepática y renal pueden modificar las concentraciones plasmáticas de los anticoagulantes, mientras que el transporte hepático por la glicoproteína $\mathrm{P}$, que está implicado en la eliminación de estos fármacos, suele verse afectado por varios agentes antineoplásicos. Aunque en la actualidad no sabemos con exactitud la relevancia clínica de estas complejas interacciones, es razonable pensar que el aumento en la concentración cerebral de los fármacos se corresponda con un riesgo hemorrágico elevado, incluso para el temido sangrado intracraneano.

Mientras el peso de la evidencia siga siendo insuficiente, el inicio de la anticoagulación en el paciente oncológico con FA seguirá siendo un gran desafío clínico que requiere, tras un correcto ba- lance de los riesgos tromboembólicos versus hemorrágicos, la selección del fármaco más adecuado, su dosificación y monitoreo estrecho. Más que nunca, estas decisiones clínicas deben tomarse en forma individualizada, integrando un equipo multidisciplinario.

\section{Carolina Artucio, ORCID: 0000-0001-9945-7171 Mónica Delorenzi, ORCID: 0000-0001-9457-7692 Mariela Lujambio, ORCID: 0000-0003-1165-175X Natalia Moreira, ORCID: 0000-0002-7589-1659 Andrea Simeone, ORCID: 0000-0002-8391-091X Andreina Gómez, ORCID: 0000-0003-1911-8730 Alexis Dini, ORCID: 0000-0001-6291-6119 Andrés Gari, ORCID: 0000-0002-8223-2707. Verónica Montauban, ORCID: 0000-0003-3813-5763 Giovanna Podstavka, ORCID: 0000-0002-9088-1432 Soledad Murguía, ORCID: 0000-0001-8135-7316 Valentina Agorrody, ORCID: 0000-0001-9386-9343 Andrea Simeone, ORCID: 0000-0002-8391-091X}

Este artículo fue aceptado para su publicación por el Editor jefe, Dr. Federico Ferrando

\section{Bibliografía}

1. Chang H, Moudgil R, Scarabelli T, Okwuosa T, Yeh E. cardiovascular complications of cancer therapy: best practices in diagnosis, prevention, and management: part 1. J Am Coll Cardiol 2017; 70(20):2536-51. doi: 10.1016/j.jacc.2017.09.1096

2. Koutsoukis A, Ntalianis A, Repasos E, Kastritis E, Dimopoulos MA, Paraskevaidis I. Cardio-oncology: a focus on cardiotoxicity. Eur Cardiol 2018; 13(1):64-9. doi: 10.15420/ecr.2017:17:2

3. Libby $\mathbf{P}$, Kobold S. Inflammation: a common contributor to cancer, aging, and cardiovascular diseases-expanding the concept of cardio-oncology. Cardiovasc Res 2019; 115(5):824-9. doi: 10.1093/cvr/ cvz058

4. Zamorano J, Lancellotti P, Rodriguez Muñoz D, Aboyans V, Asteggiano R, Galderisi M, et al; ESC Scientific Document Group. 2016 ESC Position Paper on cancer treatments and cardiovascular toxicity developed under the auspices of the ESC Committee for Practice Guidelines: the Task Force for cancer treatments and cardiovascular toxicity of the European Society of Cardiology (ESC). Eur Heart J 2016; 37(36):2768-801. doi: 10.1093/ eurheartj/ehw211

5. Libby P, Bonow R, Zipes D, Mann D, Braunwald E. Braunwald tratado de cardiología: texto de medicina cardiovascular. 8 ed. Barcelona: Elsevier, 2009.

6. Larsen C, Mulvagh S. Cardio-oncology: what you need to know now for clinical practice and echocardiography. Echo Res Pract 2017; 4(1):R33-R41. doi: 10.1530/ERP-17-0013 
7. Desai M, Jellis C, Kotecha R, Johnston D, Griffin B. Radiation-associated cardiac disease: a practical approach to diagnosis and management. JACC Cardiovasc Imaging 2018; 11(8):1132-49. doi: 10.1016/j.jcmg.2018.04.028

8. Sarkiss M, Yusuf S, Warneke C, Botz G, Lakkis N, Hirch-Ginsburg C, et al. Impact of aspirin therapy in cancer patients with thrombocytopenia and acute coronary syndromes. Cancer 2007; 109(3):621-7. doi: 10.1002/cncr.22434

9. Iliescu C, Grines C, Herrmann J, Yang E, Cilingiroglu M, Charitakis K, et al. SCAI Expert consensus statement: Evaluation, management, and special considerations of cardio-oncology patients in the cardiac catheterization laboratory (endorsed by the cardiological society of india, and sociedad Latino Americana de Cardiologia intervencionista). Catheter Cardiovasc Interv 2016; 87(5):E202-23. doi: 10.1002/ccd.26379

10. Iliescu C, Munoz E, Giza D, Marmagkiolis K. Special considerations of cardio-oncology patients in the cardiac catheterization laboratory. Washington Dc: American College of Cardiology, 2017. Disponible en: https://www.acc.org/latest-in-cardiology/articles/2017/01/30/07/44/special-considerations-of-cardio-oncology-patients-in-the-cardiac-catheterization-laboratory. [Consulta 2021].

11. Lax J, Piñeiro D. Consenso de diagnóstico, prevención y tratamiento de la cardiotoxicidad por tratamiento médico del cáncer. Rev Argent Cardiol 2013; 81(6):530-6.

12. Velders M, Boden H, Hofma S, Osanto S, van der Hoeven B, Heestermans A, et al. Outcome after ST elevation myocardial infarction in patients with cancer treated with primary percutaneous coronary intervention. Am J Cardiol 2013; 112(12):1867-72. doi: 10.1016/j.amjcard.2013.08.019

13. Iliescu C, Durand J, Kroll M. Cardiovascular interventions in thrombocytopenic cancer patients. Tex Heart Inst J 2011; 38(3):259-60.

14. Piccirillo J, Tierney R, Costas I, Grove L, Spitznagel E Jr. Prognostic importance of comorbidity in a hospital-based cancer registry. JAMA 2004; 291(20):2441-7. doi: 10.1001/ jama.291.20.2441

15. Mouhayar E, Salahudeen A. Hypertension in cancer patients. Tex Heart Inst J 2011; 38(3):263-5.

16. Chu T, Rupnick M, Kerkela R, Dallabrida S, Zurakowski D, Nguyen L, et al. Cardiotoxicity associated with tyrosine kinase inhibitor sunitinib. Lancet 2007; 370(9604):2011-9. doi: 10.1016/S01406736(07)61865-0

17. Yeh E, Bickford C. Cardiovascular complications of cancer therapy: incidence, pathogenesis, diagnosis, and management. J Am Coll Cardiol 2009; 53(24):2231-47. doi: 10.1016/j.jacc.2009.02.050

18. Fraeman K, Nordstrom B, Luo W, Landis S, Shantakumar S. Incidence of new-onset hypertension in cancer patients: a retrospective cohort study. Int J Hypertens 2013; 2013:379252. doi: $10.1155 / 2013 / 379252$
19. Vázquez H. Hipertensión arterial y tratamiento del cáncer. Rev Urug Cardiol 2018; 33(1):43-9.

20. Milan A, Puglisi E, Ferrari L, Bruno G, Losano I, Veglio F. Arterial hypertension and cancer. Int J Cancer 2014; 134(10):2269-77. doi: 10.1002/ ijc. 28334

21. Brinda B, Viganego F, Vo T, Dolan D, Fradley M. Anti-VEGF-induced hypertension: a review of pathophysiology and treatment options. Curr Treat Options Cardiovasc Med 2016; 18(5):33. doi: 10.1007/s11936-016-0452-z

22. Izzedine H, Ederhy S, Goldwasser F, Soria J, Milano G, Cohen A, et al. Management of hypertension in angiogenesis inhibitor-treated patients. Ann Oncol 2009; 20(5):807-15. doi: 10.1093/annonc/ $\operatorname{mdn} 713$

23. Syrigos K, Karapanagiotou E, Boura P, Manegold C, Harrington K. Bevacizumab-induced hypertension: pathogenesis and management. BioDrugs 2011; 25(3):159-69. doi: 10.2165/11590180000000000-00000

24. Vasudev N, Reynolds A. Anti-angiogenic therapy for cancer: current progress, unresolved questions and future directions. Angiogenesis 2014; 17(3):471-94. doi: 10.1007/s10456-014-9420-y

25. Hall P, Harshman L, Srinivas S, Witteles R. The frequency and severity of cardiovascular toxicity from targeted therapy in advanced renal cell carcinoma patients. JACC Heart Fail 2013; 1(1):728. doi: 10.1016/j.jchf.2012.09.001

26. Scartozzi M, Galizia E, Chiorrini S, Giampieri R, Berardi R, Pierantoni C, et al. Arterial hypertension correlates with clinical outcome in colorectal cancer patients treated with first-line bevacizumab. Ann Oncol 2009; 20(2):227-30. doi: 10.1093/annonc/mdn637

27. Rini B, Cohen D, Lu D, Chen I, Hariharan S, Gore M, et al. Hypertension as a biomarker of efficacy in patients with metastatic renal cell carcinoma treated with sunitinib. J Natl Cancer Inst 2011; 103(9):763-73. doi: 10.1093/jnci/djr128

28. López-Fernández T, Martín García A, Santaballa Beltrán A, Montero Luis Á, García Sanz R, Mazón Ramos P, et al. Cardio-onco-hematología en la práctica clínica: document de consenso y recomendaciones. Rev Esp Cardiol 2017; 70(6):474-86. doi: 10.1016/j.recesp.2016.12.021

29. Souza V, Silva E, Ribeiro M, Martins W. Hypertension in patients with cancer. Arq Bras Cardiol 2015; 104(3):246-52. doi: 10.5935/abc.20150011

30. Rossi G, Seccia T, Maniero C, Pessina A. Drug-related hypertension and resistance to antihypertensive treatment: a call for action. J Hypertens 2011; 29(12):2295-309. doi: 10.1097/HJH. 0b013e32834c465d

31. Maitland M, Bakris G, Black H, Chen H, Durand J, Elliott W, et al; Cardiovascular Toxicities Panel, Convened by the Angiogenesis Task Force of the National Cancer Institute Investigational Drug Steering Committee. Initial assessment, surveillance, and management of blood pressure in patients receiving vascular 
endothelial growth factor signaling pathway inhibitors. J Natl Cancer Inst 2010; 102(9):596-604. doi: 10.1093/jnci/djq091

32. Mazón P, Marín F, Cosín-Sales J, Cordero A, Roldán I, García-Moll X, et al. Comentarios a la guía ESC/ESH 2018 sobre el diagnóstico y tratamiento de la hipertensión arterial. Rev Esp Cardiol 2019; 72(2):104-8. doi: 10.1016/j.recesp.2018.11.022

33. Copur M, Obermiller A. An algorithm for the management of hypertension in the setting of vascular endothelial growth factor signaling inhibition. Clin Colorectal Cancer 2011; 10(3):151-6. doi: 10.1016/j.clcc.2011.03.021

34. Ghadri J, Wittstein I, Prasad A, Sharkey S, Dote K, Akashi Y, et al. International expert consensus document on takotsubo syndrome (part i): clinical characteristics, diagnostic criteria, and pathophysiology. Eur Heart J 2018; 39(22):2032-46. doi: 10.1093/eurheartj/ehy076

35. Joy P, Guddati A, Shapira I. Outcomes of Takotsubo cardiomyopathy in hospitalized cancer patients. J Cancer Res Clin Oncol 2018; 144(8):153945. doi: 10.1007/s00432-018-2661-1

36. Madias J. What is/are the trigger(s) of takotsubo syndrome in cancer patients receiving chemotherapy? Int J Cardiol 2016; 222:253. doi: 10.1016/j.ijcard.2016.07.246

37. Labianca R, Beretta G, Clerici M, Fraschini P, Luporini G. Cardiac toxicity of 5-fluorouracil: a study on 1083 patients. Tumori 1982; 68(6):505-10.

38. Saif M, Shah M, Shah A. Fluoropyrimidine-associated cardiotoxicity: revisited. Expert Opin Drug Saf 2009; 8(2):191-202. doi: 10.1517/14740330902733961

39. Ishitsuka H. Capecitabine: preclinical pharmacology studies. Invest New Drugs 2000; 18(4):343-54. doi: 10.1023/a:1006497231579

40. Franco T, Khan A, Joshi V, Thomas B. Takotsubo cardiomyopathy in two men receiving bevacizumab for metastatic cancer. Ther Clin Risk Manag 2008; 4(6):1367-70. doi: 10.2147/tcrm.s3960

41. Ng K, Dearden C, Gruber P. Rituximab-induced Takotsubo syndrome: more cardiotoxic than it appears? BMJ Case Rep 2015; 2015:bcr2014208203. doi: 10.1136/bcr-2014-208203

42. Desai A, Noor A, Joshi S, Kim A. Takotsubo cardiomyopathy in cancer patients. Cardiooncology 2019; 5:7. doi: 10.1186/s40959-019-0042-9

43. Giza D, López-Mattei J, Vejpongsa P, Munoz E, Iliescu G, Kitkungvan D, et al. Stress-Induced Cardiomyopathy in Cancer Patients. Am J Cardiol 2017; 120(12):2284-8. doi: 10.1016/j.amjcard.2017.09.009

44. Dominguez F, Kühl U, Pieske B, Garcia-Pavia P, Tschöpe C. Update on myocarditis and inflammatory cardiomyopathy: reemergence of endomyocardial biopsy. Rev Esp Cardiol (Engl Ed) 2016; 69(2):178-87. doi: 10.1016/j.rec.2015.10.015

45. Aretz H. Myocarditis: the Dallas criteria. Hum Pathol 1987; 18(6):619-24. doi: 10.1016/s00468177(87)80363-5
46. Friedrich M, Sechtem U, Schulz-Menger J, Holmvang G, Alakija P, Cooper L, et al; International Consensus Group on Cardiovascular Magnetic Resonance in Myocarditis. Cardiovascular magnetic resonance in myocarditis: a JACC white paper. J Am Coll Cardiol 2009; 53(17):1475-87. doi: 10.1016/j.jacc.2009.02.007

47. Abdel-Aty H, Boyé P, Zagrosek A, Wassmuth R, Kumar A, Messroghli D, et al. Diagnostic performance of cardiovascular magnetic resonance in patients with suspected acute myocarditis: comparison of different approaches. J Am Coll Cardiol 2005; 45(11):1815-22. doi: 10.1016/j.jacc.2004.11.069

48. Aletras A, Kellman P, Derbyshire J, Arai A. ACUT2E TSE-SSFP: a hybrid method for T2-weighted imaging of edema in the heart. Magn Reson Med 2008; 59(2):229-35. doi: 10.1002/ mrm.21490

49. Topalian S, Hodi F, Brahmer J, Gettinger S, Smith D, McDermott D, et al. Safety, activity, and immune correlates of anti-PD-1 antibody in cancer. N Engl J Med 2012; 366(26):2443-54. doi: 10.1056/NEJMoa1200690

50. Rittmeyer A, Barlesi F, Waterkamp D, Park K, Ciardiello F, von Pawel J, et al; OAK Study Group. Atezolizumab versus docetaxel in patients with previously treated non-small-cell lung cancer (OAK): a phase 3, open-label, multicentre randomised controlled trial. Lancet 2017; 389(10066):255-65. doi: 10.1016/S0140-6736(16)32517-X

51. Hamanishi J, Mandai M, Ikeda T, Minami M, Kawaguchi A, Murayama T, et al. Safety and antitumor activity of anti-PD-1 antibody, nivolumab, in patients with platinum-resistant ovarian cancer. J Clin Oncol 2015; 33(34):4015-22. doi: 10.1200/ JCO.2015.62.3397

52. Wolchok J, Chiarion-Sileni V, González R, Rutkowski P, Grob J, Cowey C, et al. Overall Survival with Combined Nivolumab and Ipilimumab in Advanced Melanoma. N Engl J Med 2017; 377(14):1345-56. doi: 10.1056/NEJMoa1709684

53. Lacouture M, Wolchok J, Yosipovitch G, Kähler K, Busam K, Hauschild A. Ipilimumab in patients with cancer and the management of dermatologic adverse events. J Am Acad Dermatol 2014; 71(1):161-9. doi: 10.1016/j.jaad.2014.02.035

54. Heinzerling L, Ott P, Hodi F, Husain A, Tajmir-Riahi A, Tawbi H, et al. Cardiotoxicity associated with CTLA4 and PD1 blocking immunotherapy. J Immunother Cancer 2016; 4:50. doi: 10.1186/ s40425-016-0152-y

55. Hofmann L, Forschner A, Loquai C, Goldinger S, Zimmer L, Ugurel S, et al. Cutaneous, gastrointestinal, hepatic, endocrine, and renal side-effects of anti-PD-1 therapy. Eur J Cancer 2016; 60:190209. doi: 10.1016/j.ejca.2016.02.025

56. Baban B, Liu J, Qin X, Weintraub N, Mozaffari M. Upregulation of programmed death-1 and its ligand in cardiac injury models: interaction with GADD153. PLoS One 2015; 10(4):e0124059. doi: 10.1371/journal.pone.0124059 
57. Upadhrasta S, Elias H, Patel K, Zheng L. Managing cardiotoxicity associated with immune checkpoint inhibitors. Chronic Dis Transl Med 2019; 5(1):6-14. doi: 10.1016/j.cdtm.2019.02.004

58. D’ortencio A, Navigante A. Insuficiencia cardíaca relacionada a quimioterapia: nuevo enfoque. Insuf Card 2006; 1(1):28-32.

59. Varricchi G, Galdiero M, Marone G, Criscuolo G, Triassi M, Bonaduce D, et al. Cardiotoxicity of immune checkpoint inhibitors. ESMO Open 2017; 2(4):e000247. doi: 10.1136/esmoopen-2017-000247

60. Johnson D, Balko J, Compton M, Chalkias S, Gorham J, Xu Y, et al. Fulminant myocarditis with combination immune checkpoint blockade. N Engl J Med 2016; 375(18):1749-55. doi: 10.1056/ NEJMoa1609214

61. Mahmood S, Fradley M, Cohen J, Nohria A, Reynolds K, Heinzerling L, et al. Myocarditis in patients treated with immune checkpoint inhibitors. J Am Coll Cardiol 2018; 71(16):1755-64. doi: 10.1016/j.jacc.2018.02.037

62. Moslehi J, Salem J, Sosman J, Lebrun-Vignes B, Johnson D. Increased reporting of fatal immune checkpoint inhibitor-associated myocarditis. Lancet 2018; 391(10124):933. doi: 10.1016/S01406736(18)30533-6

63. Yang S, Asnani A. Cardiotoxicities associated with immune checkpoint inhibitors. Curr Probl Cancer 2018; 42(4):422-32. doi: 10.1016/j.currproblcancer.2018.07.002

64. Brahmer J, Lacchetti C, Schneider B, Atkins M, Brassil K, Caterino J, et al; National Comprehensive Cancer Network. Management of immune-related adverse events in patients treated with immune checkpoint inhibitor therapy: American Society of Clinical Oncology clinical practice guideline. J Clin Oncol 2018; 36(17):1714-68. doi: 10.1200/JCO.2017.77.6385

65. Neilan T, Rothenberg M, Amiri-Kordestani L, Sullivan R, Steingart R, Gregory W, et al; Checkpoint Inhibitor Safety Working Group. Myocarditis associated with immune checkpoint inhibitors: an expert consensus on data gaps and a call to action. Oncologist 2018; 23(8):874-8. doi: 10.1634/theoncologist.2018-0157

66. Caforio A, Pankuweit S, Arbustini E, Basso C, Gimeno-Blanes J, Felix S, et al; European Society of Cardiology Working Group on Myocardial and Pericardial Diseases. Current state of knowledge on aetiology, diagnosis, management, and therapy of myocarditis: a position statement of the European Society of Cardiology Working Group on Myocardial and Pericardial Diseases. Eur Heart J 2013; 34(33):2636-48, 2648a-2648d. doi: 10.1093/eurheartj/eht210

67. Norwood T, Westbrook B, Johnson D, Litovsky S, Terry N, McKee S, et al. Smoldering myocarditis following immune checkpoint blockade. J Immunother Cancer 2017; 5(1):91. doi: 10.1186/ s40425-017-0296-4

68. Suter T, Ewer M. Cancer drugs and the heart: importance and management. Eur Heart J 2013;
34(15):1102-11. doi: 10.1093/eurheartj/ehs 181

69. Yuan M, Zhang Z, Tse G, Feng X, Korantzopoulos P, Letsas K, et al. Association of cancer and the risk of developing atrial fibrillation: a systematic review and meta-analysis. Cardiol Res Pract 2019; 2019:8985273. doi: 10.1155/2019/8985273

70. Farmakis D, Parissis J, Filippatos G. Insights into onco-cardiology: atrial fibrillation in cancer. $\mathrm{J}$ Am Coll Cardiol 2014; 63(10):945-53. doi: 10.1016/j. jacc.2013.11.026

71. Yun J, Choi E, Han K, Park S, Jung J, Park S, et al. Risk of Atrial fibrillation according to cancer type: a nationwide population-based study. JACC CardioOncol 2021; 3(2):221-32. doi: 10.1016/j.jaccao.2021.03.006

72. Kilickap S, Barista I, Akgul E, Aytemir K, Aksoy S, Tekuzman G. Early and late arrhythmogenic effects of doxorubicin. South Med J 2007; 100(3):2625. doi: 10.1097/01.smj.0000257382.89910.fe

73. Fradley M, Viganego F, Kip K, Martin A, Patel A, Ismail-Khan $\mathbf{R}$, et al. Rates and risk of arrhythmias in cancer survivors with chemotherapy-induced cardiomyopathy compared with patients with other cardiomyopathies. Open Heart 2017; 4(2):e000701. doi: 10.1136/openhrt-2017-000701

74. Sureddi R, Amani F, Hebbar P, Williams D, Leonardi M, Paydak H, et al. Atrial fibrillation following autologous stem cell transplantation in patients with multiple myeloma: incidence and risk factors. Ther Adv Cardiovasc Dis 2012; 6(6):229-36. doi: 10.1177/1753944712464102

75. Arun M, Brauneis D, Doros G, Shelton A, Sloan J, Quillen K, et al. The incidence of atrial fibrillation among patients with $\mathrm{AL}$ amyloidosis undergoing high-dose melphalan and stem cell transplantation: experience at a single institution. Bone Marrow Transplant 2017; 52(9):1349-51. doi: 10.1038/bmt.2017.148

76. Dimopoulos M, Goldschmidt H, Niesvizky R, Joshua D, Chng W, Oriol A, et al. Carfilzomib or bortezomib in relapsed or refractory multiple myeloma (ENDEAVOR): an interim overall survival analysis of an open-label, randomised, phase 3 trial. Lancet Oncol 2017; 18(10):1327-37. doi: 10.1016/ S1470-2045(17)30578-8

77. Nazer B, Humphreys B, Moslehi J. Effects of novel angiogenesis inhibitors for the treatment of cancer on the cardiovascular system: focus on hypertension. Circulation 2011; 124(15):1687-91. doi: 10.1161/CIRCULATIONAHA.110.992230

78. Mego M, Reckova M, Obertova J, Sycova-Mila Z, Brozmanova K, Mardiak J. Increased cardiotoxicity of sorafenib in sunitinib-pretreated patients with metastatic renal cell carcinoma. Ann Oncol 2007; 18(11):1906-7. doi: 10.1093/annonc/mdm489

79. Yun S, Vincelette N, Acharya U, Abraham I. Risk of atrial fibrillation and bleeding diathesis associated with ibrutinib treatment: a systematic review and pooled analysis of four randomized controlled trials. Clin Lymphoma Myeloma Leuk 2017; 17(1):31-37.e13. doi: 10.1016/j.clml.2016.09.010 
80. Asnani A, Manning A, Mansour M, Ruskin J, Hochberg E, Ptaszek L. Management of atrial fibrillation in patients taking targeted cancer therapies. Cardiooncology 2017; 3:2. doi: 10.1186/ s40959-017-0021-y
81. Hu Y, Liu C, Chang P, Tsao H, Lin Y, Chang S, et al. Incident thromboembolism and heart failure associated with new-onset atrial fibrillation in cancer patients. Int J Cardiol 2013; 165(2):355-7. doi: 10.1016/j.ijcard.2012.08.036 